27 | 2001

Histoire de l'enseignement du français langue

étrangère ou seconde dans le bassin méditerranéen.

Volume 1

\title{
L'enseignement du français en Egypte dans les années 1920 : une nébuleuse à plusieurs degrés de francité
}

\section{Frédéric Abécassis}

\section{(2) OpenEdition Journals}

\section{Édition électronique}

URL : https://journals.openedition.org/dhfles/2576

DOl : $10.4000 /$ dhfles. 2576

ISSN : 2221-4038

\section{Éditeur}

Société Internationale pour l'Histoire du Français Langue Étrangère ou Seconde

\section{Édition imprimée}

Date de publication : 1 décembre 2001

ISSN : 0992-7654

\section{Référence électronique}

Frédéric Abécassis, «L'enseignement du français en Egypte dans les années 1920 : une nébuleuse à plusieurs degrés de francité », Documents pour l'histoire du français langue étrangère ou seconde [En ligne], 27 | 2001, mis en ligne le 31 janvier 2014, consulté le 27 mai 2021. URL : http:// journals.openedition.org/dhfles/2576 ; DOI : https://doi.org/10.4000/dhfles.2576

Ce document a été généré automatiquement le 27 mai 2021.

(c) SIHFLES 


\title{
L'enseignement du français en Egypte dans les années 1920 : une nébuleuse à plusieurs degrés de francité
}

\author{
Frédéric Abécassis
}

1 Cette contribution vise à dresser un tableau contrasté de la francophonie égyptienne des années vingt. Ce moment peut être considéré comme un apogée. Il faut en effet poser d'emblée l'aspect contradictoire de cette période : d'une part, les promoteurs de la francophonie voient s'ouvrir dès la fin de la première guerre mondiale des perspectives de développement inespérées quelques années plus tôt. D'un autre côté, ces années constituent aussi un moment où la francophonie, très minoritaire dans le pays et résolument cantonnée aux marges de l'État, tend à s'enfermer dans un ghetto. Elle n'en sortira plus, scellant à terme dans un exil commun le sort de ceux qui y auront tout investi, en particulier leurs parcours de formation.

2 La contradiction peut se comprendre par le recours à la métaphore de la nébuleuse : il n'y a pas une mais des francophonies dans l'Égypte des années vingt. Les modalités, le degré de pratique et le niveau d'appropriation de la langue étaient tributaires du positionnement des institutions chargées de la véhiculer entre l'État français, l'État égyptien et le public qu'elles visaient. Multiplicité d'acteurs, diversité des cibles et des héritages, tout cela nous renvoie, derrière une communauté francophone qui se pensait volontiers comme une élite - un officier de marine français allait jusqu'à affirmer, lors d'un passage à Alexandrie en 1923 : « tout ce qui pense en Égypte provient de nos écoles ${ }^{1} »$ - à quelque chose qui tient à la fois du patchwork et du palimpseste - au sens où chaque "génération " francophone n'a cessé de tendre à vouloir effacer la précédente. 


\section{Les enjeux singuliers d'une Égypte indépendante}

3 L'Égypte est un État qui passe dans ces années d'une domination coloniale directe (occupation britannique de 1882, officialisée par un protectorat en 1914) à une situation d'indépendance. Certes, celle-ci demeurait formelle et relative ${ }^{2}$, mais elle n'en rendait pas moins cruciaux une série d'enjeux fondateurs autour de la définition d'une nationalité, d'une citoyenneté, d'une société politique et, en définitive, des « fondements du pouvoir ${ }^{3}$.»

4 Le cycle révolutionnaire des années 1919-1922 et l'adoption d'une constitution en 1923 mirent en place un jeu serré entre trois entités porteuses de politiques linguistiques et éducatives radicalement différentes. Les Britanniques demeuraient attachés au statu quo. Soucieux de pérenniser leur domination au delà des quatre domaines réservés, ils entendaient ne céder en rien la haute main sur l'administration aux Français ou aux Italiens, leurs rivaux.

5 Le Palais était de son côté porteur d'un projet aristocratique que l'on pourrait qualifier d'orléanisme à l'Égyptienne. Fouad $\mathrm{I}^{\mathrm{er}}$ chercha à constituer autour de lui une société politique francophone, susceptible de récolter les bénéfices d'un mouvement national dirigé contre les Anglais, tout en étant, en raison même de sa francophonie, particulièrement restreinte. Cette vision rejoignait celle des autorités diplomatiques françaises, qui se félicitaient de voir le français faire fonction de droit d'entrée dans le champ politique, et les minorités juives et chrétiennes largement francisées intégrées à l'État, comme autant de protégés (dhimmi) d'un souverain inspiré de la " grande tradition » de l'islam.

6 Le troisième projet se réclamait au contraire d'une légitimité démocratique. Il était porté par le Wafd, parti issu de la " délégation ${ }^{4}$ » conduite par Saad Zaghloul pacha, qui osa en 1918 demander au Haut-Commissaire britannique l'indépendance de l'Égypte, et cristallisa autour de lui le mouvement national égyptien. Si l'évidence de ce positionnement politique voulait que l'arabe fût la langue nationale du pays et devînt progressivement celle de l'ensemble de l'État, le positionnement social du mouvement était plus ambigu. Ses dirigeants, notables francophones souvent juristes de formation, baignaient dans des référents liés à la Révolution française et à l'idée de nation souveraine. Mais c'est seulement en arabe qu'ils pouvaient espérer mobiliser une base sociale, dans un pays massivement illettré ${ }^{5}$. Au niveau intermédiaire, les cadres de l'organisation parlaient l'arabe dialectal, lisaient l'arabe classique et écrivaient le plus souvent un arabe " médian " en usage depuis quelques décennies dans la presse et certaines administrations, qui n'avait toutefois pas encore trouvé sa codification et se nourrissait pour la terminologie scientifique d'emprunts lexicaux français, italiens ou anglais $^{6}$. Ceux qui avaient suivi un cursus dans l'enseignement "moderne ", minoritaires par rapport à ceux qui sortaient de la filière azhariste traditionnelle, avaient appris l'anglais comme langue étrangère. Plus exactement, c'était le cas de la jeune génération : formée à partir des années 1890, elle n'avait connu que l'occupation britannique et occupait du fait de son jeune âge des places subalternes dans l'administration. La génération plus ancienne, celle qui tenait des postes plus importants, était encore le témoignage vivant d'une situation antérieure où le français l'emportait sur l'anglais dans le système scolaire égyptien.

7 La mise en avant de l'anglais, du français ou de l'arabe n'était donc politiquement pas neutre, et cette cristallisation autour de l'enjeu linguistique n'était que le reflet d'une 
société où le système éducatif " moderne ", constitué depuis près d'un siècle en rupture avec le système de formation " traditionnel ", était devenu un enjeu central - on peut même aller jusqu'à dire qu'il était devenu le fondement même de la légitimité politique.

8 La vénérable université d'Al-Azhar avait assuré pendant des siècles la formation des cadres administratifs et judiciaires de l'Égypte. C'est à l'époque de Muhammad'Ali (1804-1849) que commença à se développer pour les besoins de l'État - et plus précisément de son armée - un système éducatif « moderne » à l'européenne. Ces créations entraînèrent la dépréciation rapide de l'enseignement azhariste, et rendirent sa réforme particulièrement difficile. Dès les décennies 1860-1880, quatre écoles brossaient déjà les grands traits de l'enseignement supérieur égyptien : École de médecine, École khédiviale de droit, École polytechnique et École normale (Dârel-'Olûm). En 1908, la fondation de l'Université égyptienne par un groupe de notables vint compléter l'édifice en le dotant d'une faculté des Lettres. La traduction en français du nom de ces écoles montre assez quel parallèle était établi avec les institutions françaises, et l'existence d'une paternité revendiquée sinon réellement assumée. Il reste que ces phases d'expansion de l'appareil scolaire " moderne » égyptien ont été, pour des raisons diverses, soutenues par la France, et souvent d'ailleurs liées à différents moments de promotion du français.

9 L'occupation anglaise (1882) mit un coup d'arrêt brutal à cet essor de l'appareil éducatif d'État. Pour des raisons à la fois financières et idéologiques, elle fut une période de déflation scolaire : avant 1882, on comptait en Égypte 25 écoles secondaires ; en 1906, elles n'étaient plus que 3 . Avec un budget qui n'excéda jamais $1 \%$ du budget de l'État de 1883 à 1901, et parvint péniblement à se hisser au chiffre record de 3,4\% à la veille de la guerre, l'éducation fut délibérément sacrifiée à la recherche de l'équilibre financier. Sa limitation aux classes les plus aisées était aussi conçue comme un instrument de stabilité politique. Dans l'optique d'un Lord Cromer, former des diplômés " indigènes ", c'était prendre le risque de créer une masse de " semi-occidentalisés ${ }^{7}$ », qui, frustrés dans leurs attentes professionnelles, grossiraient les rangs du mouvement nationaliste.

Il faut attendre les années 1910 pour assister à un essor du système éducatif égyptien, en partie sous l'effet de la fondation de l'Université égyptienne.

11 Les initiatives privées, celles des sociétés de bienfaisance ou des conseils provinciaux, encouragées par le ministère de l'Instruction publique aboutirent à la création de plusieurs sections secondaires dans le pays. Mais les temps avaient changé, et le français, s'il régnait très largement sur l'offre d'éducation privée étrangère, était désormais réduit à la portion congrue dans l'enseignement égyptien, loin derrière l'anglais. Au demeurant, contrairement à ce que pensaient les autorités diplomatiques françaises, la politique britannique n'était pas seule en cause dans cette élimination du français de la filière d'État.

12 Pour comprendre l'importance de la demande d'accès à l'instruction et l'ampleur des frustrations face à la langue française dans la cristallisation du mouvement national égyptien, il faut enfin replacer l'Égypte dans l'espace et les référents qui étaient les siens au début des années vingt, ceux d'une société ottomane. Des distances avec la Sublime Porte avaient certes été prises depuis le premier tiers du XIX ${ }^{e}$ siècle et n'avaient cessé de s'accuser, mais l'Égypte ne fut formellement et définitivement détachée de l'empire qu'en 1914. Elle partageait avec les autres provinces un certain nombre de structures sociales et une expérience commune de la confrontation avec l'Europe. Le statut personnel était en particulier régi par des communautés instituées, 
dont les juridictions étaient reconnues par le pouvoir central ou local. Il incombait à celui-ci de faire respecter les décisions des conseils communautaires (majalis millia), mais il n'intervenait dans les affaires internes des communautés qu'en dernière extrémité, se satisfaisant plutôt d'une fonction d'arbitrage entre elles. Comme le reste de l'empire, l'Égypte connaissait aussi les capitulations, ces exemptions juridictionnelles et fiscales s'appliquant aux ressortissants « francs » dans les Échelles du Levant. Celles-ci s'étaient progressivement muées au XIXe siècle, pour des individus ou des groupes communautaires, en véritable statut d'extraterritorialité, que les consuls européens garantissaient à leurs protégés.

Depuis les années 1860, l'Égypte faisait figure d'El-Dorado méditerranéen : terre d'asile au moment de la crise libanaise de 1860 et des crises balkaniques, elle avait tiré un profit considérable du boom du coton pendant la guerre de Sécession, puis de l'appel d'air lié à l'exploitation du canal de Suez (inauguré en 1869). De toutes les provinces de l'empire avaient convergé vers Alexandrie, Le Caire, Port-Saïd. Suez ou Ismaïlia des Grecs, des Maltais, des Arméniens ou des Levantins, mais aussi de nombreux Italiens auxquels il faut ajouter des colonies françaises ou britanniques de dimensions plus modestes. Nombre de familles avaient fait souche dans le pays et prospéré à l'abri des capitulations, d'autant que l'Égypte connaissait une situation juridique originale par rapport aux autres provinces de l'empire. Depuis la réforme judiciaire des années 1875 fonctionnaient trois Tribunaux mixtes, rendus nécessaires par d'incessants conflits de juridiction. Ils substituaient à l'omnipotence des consuls sur leurs colonies des tribunaux où siégeaient des juges de différentes nationalités, qui avaient à connaître de toutes les affaires où des intérêts étrangers étaient en jeu. Quatre langues officielles $\mathrm{y}$ étaient théoriquement pratiquées, mais l'arabe, l'italien et l'anglais étaient relégués loin derrière le français dans les enceintes du Caire, de Mansoura ou de la Cour d'appel mixte d'Alexandrie.

La France tenait en effet un rang éminent parmi les puissances capitulaires, en raison d'une tradition qui faisait de la " fille aînée de l'Église » la protectrice des catholiques d'Orient. A la vérité, les choses étaient plus ambiguës. Si l'on parlait volontiers d'un protectorat sur "les chrétiens d'Orient " en général, les exemptions fiscales et judiciaires ne concernaient en réalité que les catholiques de rite latin ${ }^{8}$. Pour les communautés catholiques de rites orientaux (Maronites, Grecs-catholiques, Coptescatholiques, Arméniens-catholiques notamment), le « protectorat religieux de la France " se limitait à une garantie de libre exercice du culte avec pour contrepartie des honneurs liturgiques rendus aux représentants de la France lors de messes dites consulaires. Ce témoignage public de reconnaissance - dans lequel les autorités françaises voyaient souvent une véritable sujétion - était lui aussi très ambigu. La liberté de conscience n'était guère menacée en Égypte, et par ailleurs, la protection des étrangers et des minorités était de fait assurée depuis 1882 par l'Angleterre. La gratitude ainsi exprimée par les communautés catholiques orientales allait moins au rôle politique de la France dans la vallée du Nil - depuis Fachoda (1898) et les accords de l'Entente cordiale (1904), ce rôle était pratiquement réduit à néant - qu'à l'importance de l'activité française dans le champ scolaire. La question des minorités confessionnelles ou étrangères, de leur intégration à la nation égyptienne ou de leur exclusion était au cœur de la question politique énoncée plus haut. Si la politique des minorités a pu se poursuivre en Égypte après la Première guerre mondiale, c'est qu'elle n'avait pas pour enjeu comme en Syrie-Liban (Riffier, 
2000), un découpage territorial, mais la pérennité de liens de type clientéliste sur des colonies ou des communautés. Et c'est dans la politique scolaire qu'elle s'est le mieux exprimée : la protection était certes synonyme pour un petit nombre de familles gravitant autour des consulats - nombre plus limité qu'on croit -, de garanties juridiques ou fiscales, mais elle signifiait surtout, pour la plupart des minoritaires, la possibilité d'une promotion sociale par l'école.

Plus largement, on voit que le français était porté dans l'Égypte du début des années vingt par tout un ordre juridique et social et une conjoncture politique particulière. La perception de la France comme un soutien possible face aux Anglais, la présence de notables francophones à la tête du Wafd laissèrent libre cours à l'idée que « l'Égypte, depuis un siècle [était], en Orient, la fille aînée de la France ${ }^{9}$ ", qu'elle lui devait, depuis l'époque de Muhammad'Ali, sa renaissance intellectuelle et politique. Au delà du recours captieux à la mémoire de l'Égypte des khédives, l'enjeu central de la vie politique et sociale demeura, tout au long de l'histoire de l'Égypte dite libérale (1919-1952) l'accès à l'instruction. En grande partie confondu avec l'accès au français, il légitimait les pouvoirs politiques qui le finançaient, et on pourrait avancer, sans forcer le trait, qu'il était devenu le ressort même du lien national ou communautaire.

\section{L'héritage d'une triple tradition}

Dans les années vingt, le français d'Égypte conjuguait l'héritage d'institutions issues d'une tradition d'État, d'une tradition catholique et d'une tradition laïque. Cette capacité des institutions de diffusion du français à être en prise sur l'ensemble du système scolaire, à la fois étranger, gouvernemental, privé et communautaire était liée à la centralité des Tribunaux mixtes et de l'École française de droit dans les champs judiciaire et scolaire. Seul établissement d'enseignement d'Égypte à être entièrement financé par le Quai d'Orsay, cette dernière institution était née du passage de l'École khédiviale de droit d'une direction française à une direction britannique en 1891. De même que l'Institut français d'archéologie orientale, créé à la même époque, devait être l'antichambre française du Service des Antiquités égyptiennes, l'École française de droit - où l'on pouvait, sans quitter l'Égypte, décrocher une licence de la Faculté de droit de Paris - était vouée à fournir aux Tribunaux mixtes des juristes francophones.

Véritable clé de voûte du système, l'École française de droit avait un recrutement très diversifié. Il s'étendait jusqu'à l'université d'Al-Azhar, grâce à une école de l'Alliance française qui délivrait des cours du soir à certains étudiants. Cette "École Al-Azhar » était le seul établissement scolaire d'Égypte directement et totalement géré par le comité du Caire de l'Alliance française ${ }^{10}$. Ce recrutement sur concours d'étudiants musulmans de milieux plutôt modestes, arrachés à un cursus considéré comme " médiéval » (Reid, 1991 : 15) ne fournissait pas aux Tribunaux mixtes ses magistrats - les Égyptiens n'avaient d'ailleurs accès qu'au Parquet - ni même la plupart de ses avocats. Il était surtout destiné à pourvoir des postes subalternes (greffiers, huissiers...) en personnels reconnaissants et dévoués à la France. Il reste que la filière azhariste de l'École française de droit n'était plus qu'une survivance. Tout comme celle qui permettait d'y accéder après de simples études secondaires, elle court-circuitait les baccalauréats égyptien et français, alors que l'on pouvait passer l'un et l'autre en Égypte. C'est la raison pour laquelle le concours local d'accès à l'École fut remis en cause dès le début des années vingt, pour disparaître définitivement en 1933. 
Le français avait été porté, au cours du siècle précédent, par un enseignement d'État mis à mal par l'occupation britannique. La décennie 1920-1930 fut celle d'une résurrection - les effectifs doublèrent pratiquement entre ces deux dates - et d'un retour sensible du français dans le cycle secondaire égyptien. La réforme, initiée par certaines institutions privées égyptiennes avec l'appui de l'Alliance française ${ }^{11}$, fut portée dans l'enseignement gouvernemental par des hommes politiques proches du Palais. En augmentant le nombre d'heures consacrées au français, ils répondaient à une demande sociale, affichaient leur opposition aux Anglais et donnaient satisfaction au souhait du Quai d'Orsay de voir la diffusion du français s'auto-financer en Égypte.

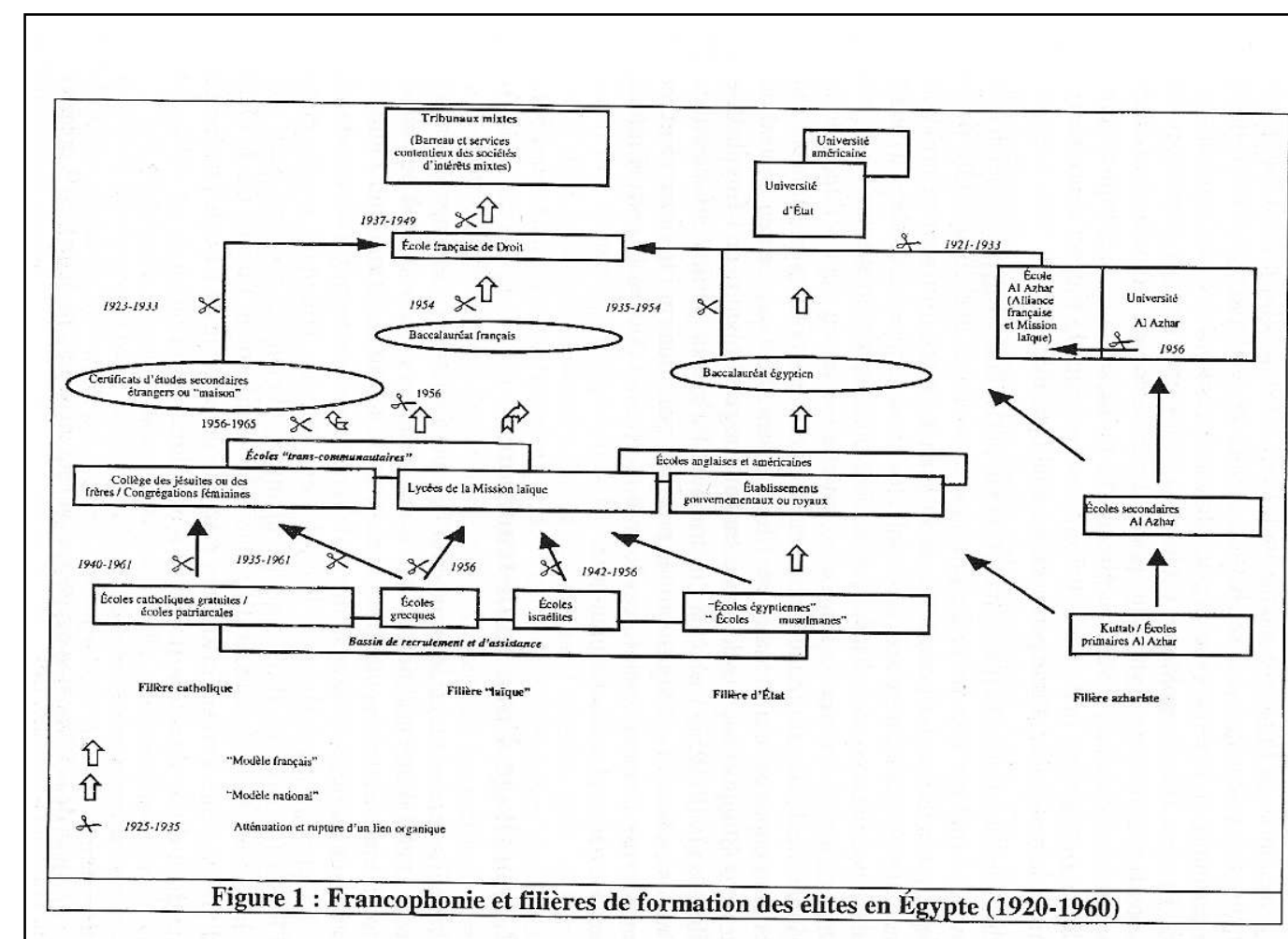

C'est dans l'enseignement supérieur que le retour du français fut beaucoup plus difficile. L'Université égyptienne devint université d'État en 1925 et entreprit de réunir en autant de facultés les différents établissements supérieurs existants. Toute la décennie fut marquée par une lutte d'influence entre la France, l'Italie et l'Angleterre pour obtenir des chaires dans les facultés de lettres et de droit. Cette dernière était en concurrence directe avec l'École française de droit, et la pression du Palais pour faire du champ judiciaire un cénacle francophone heurtait cette fois de front les aspirations et surtout les qualifications des étudiants. Nationalistes et Britanniques purent ainsi s'ériger en défenseurs de l'anglais et de l'arabe pour faire barrage au français. Ce choix linguistique, qui était aussi un choix de préférence nationale, consacrait l'élimination à terme des minorités juives et chrétiennes francophones de la société politique.

21 En effet, la diffusion et le statut privilégié du français étaient aussi l'héritage d'une tradition confessionnelle plus récente, qui s'était épanouie précisément à partir des années 1880 : partie comblé le vide éducatif créé par la banqueroute de l'État et la politique de l'occupant. Elles offraient même, avec le français, un accès à des carrières 
dans le commerce et la finance bien plus prestigieuses et lucratives que les fonctions subalternes de l'administration réservées aux diplômés de l'enseignement égyptien.

Ces missions reposaient sur un triple souci de la papauté : ramener les coptes orthodoxes « au vrai bercail, qui est celui de Saint Pierre » (Mayeur, $1992: 265$ ) lutter contre les missions protestantes américaines, très actives en Haute-Egypte, assurer enfin l'encadrement des fidèles catholiques latins, et surtout des églises uniates. Leur action avait rencontré, depuis la deuxième moitié du XIXe siècle, les aspirations des autorités françaises, dont le soutien ne s'est jamais démenti, même au plus fort des politiques anticléricales. Il s'agissait pour elles d'asseoir la protection française sur l'ensemble des catholiques orientaux, d'arracher à l'Autriche-Hongrie la protection de l'Église copto-catholique, et d'étendre le champ de la francophonie, conçue dès cette époque comme un marché francophone.

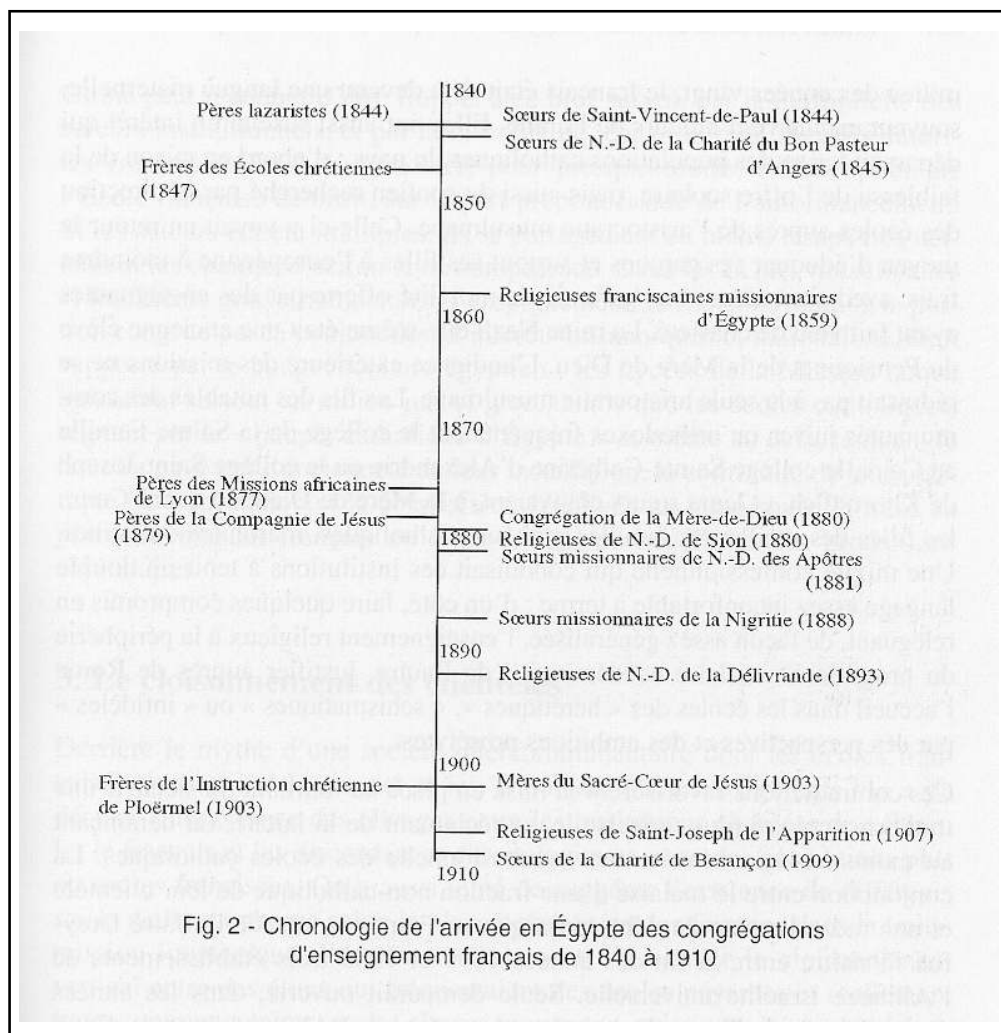

Les institutions missionnaires ont généré une francophonie très distincte de celle de la filière gouvernementale, au sens où elle était pratiquement autant féminine que masculine : dans les communautés catholiques d'Égypte, au milieu des années vingt, le français était déjà devenu une langue maternelle, souvent exclusive d'ailleurs de l'arabe. Elles ont aussi suscité un intérêt qui dépassait les seules populations catholiques du pays : d'abord en raison de la faiblesse de l'offre scolaire, mais aussi du soutien recherché par la direction des écoles auprès de l'aristocratie musulmane. Celleci y voyait en retour le moyen d'éduquer ses garçons et surtout ses filles à l'européenne à moindres frais, avec par ailleurs une garantie de moralité offerte par des enseignantes ayant fait vœu de chasteté. La reine Nazli elle-même était une ancienne élève du Pensionnat de la Mère de Dieu. L'audience extérieure des missions ne se réduisait pas à la seule aristocratie musulmane. Les fils des notables des communautés 
juives ou orthodoxes fréquentaient le collège de la Sainte-Famille au Caire, le collège Sainte-Catherine d'Alexandrie ou le collège Saint-Joseph de Khoronfish, et leurs sœurs côtoyaient, à la Mère de Dieu, au Sacré-Cœur, les filles des meilleures familles grecquescatholiques, maronites ou latines. Une mixité confessionnelle qui conduisait ces institutions à tenir un double langage assez inconfortable à terme : d'un côté, faire quelques compromis en reléguant, de façon assez généralisée, l'enseignement religieux à la périphérie du programme scolaire académique; de l'autre, justifier auprès de Rome l'accueil dans les écoles des " hérétiques ", "schismatiques » ou " infidèles " par des perspectives et des ambitions prosélytes.

Ces contradictions favorisèrent la mise en place au tournant du siècle d'une tradition scolaire plus universelle, se réclamant de la laïcité, ou dénonçant au moins la vision étroitement confessionnelle des écoles catholiques. La conjonction entre le malaise d'une fraction non-catholique de leur clientèle et une radicalisation du débat politique en France autour de l'affaire Dreyfus fit naître entre la fin des années 1890 et 1905 trois établissements de l'Alliance israélite universelle. Seule demeurait ouverte, dans les années vingt, l'école de Tanta (Delta). Les deux écoles d'Alexandrie et du Caire avaient subi de plein fouet la concurrence de deux lycées ouverts par la Mission laïque en 1909 et 1910. L'association s'était brusquement trouvée propulsée en première ligne de la politique scolaire du Quai d'Orsay au lendemain de la crise qui avait conduit à la Séparation. En l'envoyant en Égypte où la question scolaire devenait d'actualité, le Département espérait bien restaurer la tradition d'État et attirer un public de notables musulmans. Mais tout au long des années vingt, les lycées du Caire et d'Alexandrie virent leur recrutement cantonné dans la classe moyenne aisée juive et grecque orthodoxe. Le cœur de cible manquait à l'appel, mais la prospérité et la vitalité de ces deux communautés firent de ces établissements des fleurons de l'enseignement français en Orient.

On ne peut manquer d'être frappé, à ce bref survol, par la multiplicité des acteurs institutionnels, et par la diversité de leur proximité avec les autorités françaises. Et il faut insister, pour presque toutes, à l'exception de l'École française de droit, sur la part prépondérante de l'autofinancement. Si les acteurs étaient multiples, ils se partageaient en même temps très nettement les champs d'action et de compétence. Dans les Écoles musulmanes et les filières gouvernementales, l'enseignement du français, réduit à la portion congrue par la conjonction d'intérêts britanniques et nationaliste, était supporté par le budget scolaire égyptien ; les Lycées de la Mission laïque agissaient surtout en milieu juif et grec, tandis que les écoles catholiques, régnant sur le " peuple catholique d'Égypte » et même au delà, tenaient le haut du pavé par l'ancienneté de leur installation, la continuité de leur politique de francisation et la perspective, réactivée un temps avec la mise en place du mandat français en SyrieLiban, de bénéficier des protections capitulaires.

\section{Le cloisonnement des clientèles}

Derrière le mythe d'une société intercommunautaire dont les écoles françaises auraient été le creuset et les Tribunaux mixtes les garants, on a pu déceler l'importance des clivages entre les institutions chargées de véhiculer le français et les discordances chronologiques entre des générations plus ou moins francisées. On a aussi tenté de suggérer l'existence de différents styles de francophonie selon la place occupée par les 
femmes dans la transmission linguistique. Il convient également d'y ajouter le cloisonnement vertical entre des élites qui fréquentaient des écoles payantes et réellement trans-communautaires, et des classes moyennes, voire populaires, qui bénéficiaient des structures d'encadrement ou d'assistance communautaires. Si les lycées français et les collèges des jésuites ou des frères des Écoles chrétiennes étaient la vitrine d'une cohabitation harmonieuse entre notables, ils ne doivent pas être pris comme le reflet d'une société où le français aurait été porteur de modernité politique ou d'une aspiration démocratique. Ce qui était en jeu derrière la diffusion du français, c'est la pérennité de la structure communautaire et celle du pouvoir des notables sur leur communauté.

Du point de vue des institutions, une école secondaire payante permettait de financer une école élémentaire gratuite ou la scolarité d'un nombre plus ou moins élevé de boursiers. De ce point de vue, la Mission laïque et les collèges religieux fonctionnaient de façon parallèle, avec chacun son bassin d'assistance et de recrutement privilégié. Le collège de la Sainte-Famille du Caire finançait la mission jésuite en Moyenne Égypte. A Alexandrie, en 1929, les frères des Écoles chrétiennes dirigeaient neuf établissements. Selon les quartiers, ils ne s'adressaient pas au même public. Quatre collèges délivraient un enseignement secondaire. Aucun n'admettait d'élèves à titre gratuit, pas plus d'ailleurs que les écoles primaires qui leur servaient d'antichambre. Les cinq établissements restants accueillaient gratuitement un millier d'élèves, soit un peu moins du tiers du total.

Les Frères des Écoles chrétiennes accordaient ces exonérations de façon sélective et dégressive selon une échelle de proximité spirituelle évidente. Ainsi, à Alexandrie en 1929, les communautés catholiques étaient les seules à bénéficier d'un nombre d'élèves admis à titre gratuit supérieur à la moyenne

\begin{tabular}{|l|l|}
\hline Origine confessionnelle (100\%) & Élèves admis gratuitement (\%) \\
\hline Catholiques latins & $47 \%$ \\
Coptes catholiques & $45 \%$ \\
Catholiques orientaux & $33 \%$ \\
Moyenne toutes confessions & $30 \%$ \\
Orthodoxes (grecs et arméniens) & $26 \%$ \\
Coptes orthodoxes & $18 \%$ \\
Protestants & $18 \%$ \\
Musulmans & $14 \%$ \\
Juifs & $11 \%$ \\
\hline
\end{tabular}

Même dans les écoles payantes, les précautions prises pour éviter que juifs et catholiques ne se mélangent, conformément au souhait de la Propagande ${ }^{12}$, apparaissent clairement dans les deux collèges installés dans des quartiers voisins. Seuls quatre élèves juifs fréquentaient le collège de Attarine (2\%) alors que celui de Muharram Bey en accueillait 70, soit 30\% de l'effectif. Dans ce quartier où la population étrangère avait pratiquement doublé entre 1917 et 1927 (Ilbert, 1996 : 797), le collège des Frères accueillait $84 \%$ de non-catholiques soit une proportion voisine de celle du 
Lycée de la Mission laïque ${ }^{13}$. Contrairement aux autres collèges des Frères, la majorité des enseignants y était de nationalité française, ce qui devait lui valoir une bonne réputation; et il ne fait pas de doute qu'avec ses 237 élèves, il devait contribuer pour une part non négligeable au financement de l'effort scolaire de l'ensemble de la congrégation à Alexandrie.

30 Les classes sociales ne se mélangeaient pas sur les bancs des écoles françaises d'Égypte. Et les confessions, de leur côté, se mêlaient moins qu'on ne pouvait le prétendre. Du point de vue maintenant de leur public, un traitement des statistiques scolaires ${ }^{14}$ permet de distinguer trois modes bien distincts de présence dans les institutions d'enseignement du français.

31 Le mode de la défiance, que l'on pourrait aussi désigner comme un mode de résistance nationale au " rayonnement " des institutions françaises se traduit par l'usage sexuellement différencié que l'on a pu faire de ces écoles. Il était repérable non seulement chez les Égyptiens, mais chez les Italiens et les Grecs, les Allemands ou encore les musulmans et les coptes orthodoxes du discours statistique : une fille pouvait apprendre le français, et on était prêt à payer pour cela, mais le garçon devait demeurer dans la filière " nationale " constitutive de son "identité " ou plus exactement de l'investissement que l'on faisait sur ses solidarités futures.

Le mode de l'adhésion, au contraire, consistait à envoyer ses garçons et ses filles dans des institutions françaises, et à être prêt à en payer le prix. On est ici dans le monde de l'enseignement secondaire, des classes aisées, qui n'est pas sans rappeler l'Alexandrie décrite par Lawrence Durrell, empreinte de nostalgie. Les statistiques consulaires font nettement relever les communautés juives et grecques catholiques de ce profil. C'est oublier que tous les notables participaient de ce même comportement, et que par ailleurs, ces deux communautés avaient organisé par elles-mêmes, sans appui - ni donc comptabilité - du consulat français, leur propre réseau scolaire d'assistance : contrairement aux illusions des diplomates français, ce mode d'adhésion n'était en rien une allégeance à la France, mais un fonctionnement communautaire dans lequel la légitimité des dirigeants se mesurait à l'aune de leur capital social et de leur capacité à mettre l'éducation à la portée du plus grand nombre.

Le mode de la déférence était, dès lors, le symétrique exact de celui de l'adhésion : si l'on mettait bien ses garçons et ses filles dans des filières francophones, c'était dans un enseignement de type primaire voire professionnel, en étant redevable de cette scolarisation à des autorités instituées qui en assuraient la gratuité. Toutes participaient de cette légitimation par l'école : conseils communautaires, fondations royales ou sociétés de bienfaisance, jusqu'à l'État et son réseau public. Ce mode de la déférence prolongeait dans l'ordre social les bienfaits que la protection capitulaire était censée assurer dans l'ordre politique, et on ne sera pas surpris de trouver, parmi les premiers bénéficiaires des largesses de la France charitable en Égypte ${ }^{15}$ les communautés catholiques latines - notamment maltaise - et orientales.

Au demeurant, ces différentes attitudes par rapport aux écoles et à la langue françaises dépendaient sans doute davantage d'itinéraires familiaux et personnels que d'une quelconque appartenance communautaire. Peut-être une illusion d'optique statistique nous fait-elle considérer une telle appartenance comme vouée à induire certains comportements alors qu'un renversement de la proposition est certainement tout aussi pertinent : en Moyenne Égypte, où la communauté copte-catholique était une création récente des missions franciscaines puis jésuites (Martin, 1990), est-ce qu'on envoyait 
ses garçons et ses filles dans une école jésuite gratuite parce qu'on était coptecatholique, ou bien n'était-on pas de facto considéré comme copte-catholique dès lors qu'on mettait ses enfants dans une telle école, parce qu'elle était gratuite ? Les statistiques de scolarisation demeurent des discours tenus par les institutions sur leur public, selon une terminologie qui était la leur. Si elles renvoient sans doute dans la plupart des cas à un statut personnel clairement défini, elles ne permettent en rien de mesurer le degré d'identification d'un individu à sa communauté ni à sa nationalité - a fortiori lorsqu'il disposait de plusieurs passeports. Le fait que les autorités instituées, des écoles aux organismes de statistique d'État en passant par les consulats, les patriarcats et les conseils communautaires n'aient cessé de vouloir compter leurs membres ne nous dit rien sur le degré d'allégeance que des familles ou des individus pouvaient entretenir avec elles.

La francophonie en Égypte épousait très largement les clivages communautaires ou confessionnels, mais elle contribuait aussi à les construire et à les reformuler en des termes qui présupposaient, à partir d'une appartenance communautaire, une plus ou moins grande proximité à la France ou à l'Égypte. La politique française de promotion d'une nouvelle francophonie auprès de publics musulmans et coptes, le fait que des classes moyennes juives ou grecques orthodoxes se soient trouvées « chez elles » dans les lycées de la Mission laïque furent perçus par le monde de la vieille francophonie catholique comme une menace. Et elles l'exprimèrent, de façon significative, sur la thématique des fils aînés ou puînés de la France.

Les barrages institutionnels qui se mirent en place, rompant les passerelles d'une filière à l'autre se situent à la convergence de logiques nationales françaises et égyptiennes en gestation dans les années vingt, qui trouvèrent leur plein épanouissement dans les années de la grande crise. Dans un cas, il s'agissait de faire cesser en métropole la concurrence des "métèques "; dans l'autre, d'une profonde méfiance vis à vis d'un monde d'égyptianisés de fraîche date, francophones ayant en outre le plus profond mépris pour les arabophones. Ces logiques nationales travaillaient également le réduit francophone. Les décennies suivantes furent celles d'un repli communautaire qui contribuait à accroître la visibilité des institutions à mesure que la cohésion des groupes s'effilochait. La tragédie de 1956 se solda pour beaucoup par un exil en Europe, en Israël ou dans les pays neufs ; elle marqua aussi le terme d'une francophonie égyptienne qui estimait qu'elle avait naturellement vocation à tenir le haut du pavé.

\section{BIBLIOGRAPHIE}

ABDERRAZIQ, Ali (1994) : L'islam et les fondements du pouvoir. Paris, La Découverte. [Réimpression, édition originale : Le Caire, 1925].

ABECASSIS,Frédéric (2000) : L'enseignement étranger en Égypte et les élites locales, 1920-1960, Francophonie et identités nationales. Doctorat de l'université d'Aix-Marseille I.

ILBERT, Robert (1996) : Alexandrie, 1830-1930. Le Caire, IFAO. 
MARTIN, Maurice (1990) : « Les coptes catholiques, 1880-1920», Proche-Orient chrétien.

MAYEUR, Catherine (1992) : « Un collège jésuite l'ace à la société multiconfessionnelle égyptienne : la Sainte-Famille du Caire (1879-1919) », Revue d'Histoire de l'Église de France, t. LXXVII1.

RIFFIER, Jean (2000) : Les œuvres françaises en Syrie, 1860-1925. Paris, L'Harmattan, collection « Comprendre le Moyen-Orient ».

REID,Donald Malcom (1991) : Cairo University and the making of Modern Egypt. Le Caire, AUC Press. [Réimpression, édition originale : Cambridge University Press, 1990].

\section{NOTES}

1. Archives diplomatiques du Quai d'Orsay, Série K-Afriquc, sous-série Egypte, nº, p. 34-69. Rapport du 1er décembre 1921 du contre-amiral Grandclément au ministre de la Marine.

2. Aux termes de la déclaration unilatérale d'indépendance du 28 février 1922, les Britanniques réservaient quatre domaines à une négociation ultérieure avec le gouvernement égyptien: la sécurité des communications de l'Empire, la défense du pays, la protection des intérêts des étrangers et des minorités dans le pays, et la question du Soudan.

3. On se réfèrera au débat suscité par l'ouvrage de Ali Abderraziq (1925).

4. Le terme « wafd» signifie en arabe « délégation ».

5. Donald M. Reid (1991 : 105) situe l'illettrisme à $96 \%$ pour les femmes et à $76 \%$ pour les hommes à la fin des années 1920. Encore des progrès sensibles ont-ils été réalisés par rapport au début du siècle : en 1897 , le pourcentage des nationaux sachant lire et écrire est estimé à $8 \%$ pour les hommes, $0,2 \%$ pour les femmes.

6. L'Académie de langue arabe ne fut instituée par le roi Fouad qu'en 1932.

7. L'expression est de Lord Cromer lui-même

8. Et encore cette situation, issue du droit capitulaire, qui avait formellement été reconnue à la France au traité de Berlin (1878), était-elle remise en cause depuis la deuxième moitié du XIXe siècle par l'Italie, l'Allemagne ou l'Autriche, qui considéraient que la protection de leurs ressortissants (y compris les missionnaires religieux latins) leur revenait.

9. Appel de la Délégation égyptienne au Parlement français, juillet 1919, archives du Foreign Office, $407-185$.

10. On rappellera que la politique de l'Alliance française consistait d'ordinaire à subventionner des écoles existantes, non à les administrer directement. Il est vrai qu'au Caire, le président du Comité de l'Alliance française n'était autre que le directeur de l'École française de droit.

11. La politique des «écoles musulmanes » consistait à payer une partie du salaire d'enseignants de français dans des écoles privées égyptiennes musulmanes (ou coptes). Les fonds venaient du ministère, mais ils étaient versés par les comités locaux de l'Alliance française pour ne pas heurter de susceptibilités. Il s'agissait en réalité d'un investissement, destiné à « réamorcer la pompe " de renseignement français dans l'enseignement gouvernemental égyptien. Les fluctuations monétaires et la réussite partielle de l'objectif limitèrent cette politique aux années vingt. En 1923, la subvention du service des Euvres françaises dans la circonscription consulaire s'élevait à $50 \mathrm{~F}$ par élève contre $5 \mathrm{~F}$ par élève dans les écoles catholiques. On voit ainsi que la conquête d'une nouvelle clientèle avait dix fois plus de prix que l'entretien de l'ancienne.

12. II s'agit de la congrégation romaine pour la propagation de la foi, dont dépendaient les missions en Orient.

13. Cela alors que les catholiques représentaient près de la moitié (47\%) des effectifs des écoles des Frères à Alexandrie 
14. Traitement par analyse factorielle des correspondances des statistiques de fréquentation scolaire de la circonscription consulaire d'Alexandrie en 1929 (Archives diplomatiques de Nantes, série Euvres françaises, vol. 178), selon les variables du sexe, de la gratuité, de la communauté nationale ou religieuse d'origine, et du degré d'enseignement (primaire, secondaire ou professionnel).

15. L'expression est de l'historien François Charles Roux, dans un article de 1907 (Archives diplomatiques de Nantes).

\section{RÉSUMÉS}

Accédant à l'indépendance en I922, l'Égypte hérite du XIXe siècle une tradition francophone solidement ancrée. Mais discordances chronologiques et cloisonnements des clientèles interdisent alors la construction d'une véritable communauté francophone.

Achieving independence in 1922, Egypt inherited from the XIXth century a firmly anchored French-speaking tradition. But chronological discordances and the compartmentalization of the various publics in the educational institutions prevented the construction of a real Frenchspeaking community.

INDEX

Mots-clés : Francophonie, Égypte, XIXe siècle, XXe siècle

Keywords : French-speaking community, Egypt, XIXth century, XXth century

\section{AUTEUR \\ FRÉDÉRIC ABÉCASSIS}

ENS Lettres et Sciences humaines 